\title{
Envolvimento de equipes da Atenção Básica à Saúde no Controle da Tuberculose*
}

\author{
INVOLVEMENT OF HEALTH PRIMARY CARE TEAMS IN THE CONTROL OF TUBERCULOSIS
}

\author{
INVOLUCRAMIENTO DE LOS EQUIPOS DE ATENCIÓN BÁSICA A \\ LA SALUD EN EL CONTROL DE LA TUBERCULOSIS
}

\author{
Aline Aparecida Monroe ${ }^{1}$, Roxana Isabel Cardozo Gonzales ${ }^{2}$, Pedro Fredemir Palha ${ }^{3}$, \\ Cinthia Midori Sassaki ${ }^{4}$, Antonio Ruffino $\mathrm{Netto}^{5}$, Silvia Helena Figueiredo Vendramini ${ }^{6}$, \\ Tereza Cristina Scatena Villa ${ }^{7}$
}

\section{RESUMO}

Este estudo objetivou analisar o envolvimento de equipes da Atenção Básica à Saúde nas ações de controle da tuberculose, ante a percepção dos coordenadores do Programa de Controle da Tuberculose de nove municípios prioritários do Estado de São Paulo. Trata-se de uma pesquisa qualitativa, cujos dados foram coletados em junho/2005 por meio de entrevista semiestruturada com nove coordenadores e analisados pela técnica de análise de conteúdo-modalidade temática. Os resultados apontaram dificuldades para incorporação das ações de controle da tuberculose, na atenção básica, relacionadas à debilidade quantitativa e qualitativa de recursos humanos e à visão centralizada e fragmentada da organização dessas ações no sistema de saúde. A integração das atividades de controle da tuberculose na atenção básica será possível mediante organização do sistema de saúde, seguindo os princípios da atenção primária e elaboração/implementação de uma política de recursos humanos que garanta formação e capa-citação contínua das equipes de saúde.

\section{DESCRITORES}

Tuberculose.

Terapia diretamente observada.

Recursos humanos em saúde.

Atenção primária à saúde.

Enfermagem em saúde pública.

\begin{abstract}
This study was aimed at analyzing the involvement of Health Primary Care teams in the tuberculosis control actions in the perception of the Tuberculosis Control Program coordinators of nine priority municipalities of the State of São Paulo. It is a qualitative research whose data were collected in June of 2005 through semistructured interviews with nine coordinators. The content thematic modality was used for the analysis of the data. The results pointed out to difficulties in the implementation of the tuberculosis control actions in primary care related to quantitative and qualitative deficiencies of human resources and to a centralized and fragmented view regarding the organization of these actions in the health system. The integration of tuberculosis control activities in primary care is possible provided the system is organized according to primary care principles and a policy of human resources that ensures continuous education and capacity-building of health teams is elaborated/implemented.
\end{abstract}

\author{
KEY WORDS \\ Tuberculosis \\ Directly observed therapy. \\ Health manpower. \\ Primary health care. \\ Public health nursing.
}

\begin{abstract}
RESUMEN
\author{
DESCRIPTORES \\ Tuberculosis. \\ Directly observed therapy. \\ Health manpower. \\ Primary health care. \\ Public health nursing.
}

En este estudio se tuvo por objetivo analizar el involucramiento de los equipos de Atención Básica a la Salud en las acciones de control de la tuberculosis, a partir de la percepción de los coordinadores del Programa de Control de la Tuberculosis de nueve municipios prioritarios del Estado de Sao Paulo. Se trata de una investigación cualitativa, cuyos datos fueron recolectados en junio/2005 por medio de una entrevista semi-estructurada realizada a nueve coordinadores y analizados por la técnica de análisis de contenido-modalidad temática. Los resultados apuntaron dificultades para la incorporación de las acciones de control de latuberculosis, en la atención básica, relacionadas a la debilidad cuantitativa y cualitativa de recursos humanos y a la visión centralizada y fragmentada de la organización de esas acciones en el sistema de salud. La integración de las actividades de control de la tuberculosis en la atención básica será posible mediante la organización del sistema de salud, siguiendo los principios de la atención primaria y elaboración/implementación de una política de recursos humanos que garantice la formación y capacitación continua de los equipos de salud. 


\section{INTRODUÇÃO}

O Brasil ocupa o 160 lugar entre os 22 países responsáveis por $80 \%$ dos casos estimados de tuberculose (TB) no mundo e é prioritário para a implementação e expansão da estratégia DOTS (Direcly Observed Therapy Short Course - Tratamento Diretamente Observado de Curta Duração) para o controle da doença ${ }^{(1)}$. Atualmente, as políticas de saúde consideram esse controle como responsabilidade dos municípios brasileiros e reconhecem as ações do Programa de Controle da Tuberculose (PCT) como competência da Atenção Básica à Saúde (ABS) ${ }^{(2-3)}$ para melhoria do acesso às ações de diagnóstico e tratamento da doença ${ }^{(1)}$.

Nesse contexto de reforma do setor saúde, caracterizado pela descentralização de ações e serviços, a adequação quantitativa e qualitativa de recursos humanos (RH) representa um componente essencial do sistema de saúde para a prevenção e gerenciamento das condições crônicas, dentre elas, a TB. As mudanças na complexa situação global de saúde estão ocorrendo em um contexto no qual a força de trabalho não está preparada para enfrentá-las(4). A falta de envolvimento, sensibilização, conscientização e habilidade técnica; a rotatividade dos membros das equipesno sistema de saúde; a drenagem dos profissionais com maior capacitação para os sistemas privados são problemas enfrentados pelo Sistema Único de Saúde ${ }^{(5-6)}$. Programas de saúde específicos, como o da TB, reconhecem que o controle da doença ainda não foi alcançado devido à deficiência quanti-qualitativa na força de trabalho em saúde(7).

Desde 0 ano de 2003, a Organização M undial de Saúde (OMS) vem demonstrando sua preocupação em relação à qualificação das equipes de saúde para assumirem as responsabilidades com as ações de controle da TB, questão que emerge no bojo da descen-tralização e integração do controle da doença nos serviços de Atenção Primária à Saúde e que representa um dos desafios para a expansão sustentável da estratégia DOTS e para o alcance das metas de detecção e cura ${ }^{(1,8-10)}$.

Frente à relevância dos $\mathrm{RH}$ para controle da TB, o objetivo desta pesquisa foi analisar o envolvimento de equipes da Atenção Básica à Saúde com as atividades técnicas da estratégia DOTS, principalmente o Tratamento Supervisionado (TS) e a Busca de Sintomáticos Respiratórios (BSR) em municípios prioritários do Estado de São Paulo, a partir da percepção dos coordenadores do PCT.

Para compreender as principais atribuições e desafios lançados às equipes da ABS na prevenção e gerenciamento da TB, utilizou-se como marco teórico a organização da atenção às condições crônicas, com base na integração dos componentes estruturais do sistema de saúde, proposto pela OM Sem 2003, tendo como elemento de análise as atribuições designadas ao nível M icro, ou seja, a necessidade de equipes de saúde preparadas, motivadas e sensibilizadas para assumirem responsabilidades compatíveis com suas capacidades profissionais ${ }^{(5)}$. Destaca-se a influência e responsabilidade do nível Macro, no papel dos gestores municipais de saúde, referente ao adequado desenvolvimento e alocação de RH através de investimentos na formação e capacitação; do nível Meso, no papel dos coordenadores do PCT, referente à integração e co-responsabilização entre os diversos pontos de atenção à saúde, incentivos aos profissionais, garantia de equipes qualificadas para o manejo das condições crônicas, dentre elas a TB.

Este estudo parte do pressuposto que a implantação e sustentabilidade das atividades da estratégia DOTS na ABS exige integração entre os níveis M acro, M eso e M icro do sistema de saúde para garantir um contexto favorável com RH qualificados para o adequado manejo da TB.

\section{MÉTODO}

Neste trabalho optou-se pela utilização da abordagem qualitativa com a finalidade de captar a dinâmica e a complexidade da descentralização das ações de controle da TB e a assunção de responsabilidades pelas equipes que atuam em Unidades Básicas de Saúde (UBS), Programas de Saúde da Família (PSF) e Programas de Agentes Comunitários de Saúde (PACS).

0 estudo foi realizado em municípios considerados como prioritários para a intensificação das ações de controle da TB e implantação da estratégia DOTS no Estado de São Paulo. Este Estado apresenta o maior contingente de casos de TB do Brasil (cerca de 21.000 notificações anuais); coeficiente de incidência de 43,9 casos/100.000 hab.; taxas de cura próximasa $75 \%$ e abandono em torno de $10 \%(11)$.

Para a escolha dos municípios selecionados, utilizouse 0 critério técnico baseado na divisão operacional do Estado em regiões, o qual foi elaborado pela Divisão de TB do Centro de Vigilância Epidemiológica Prof. Alexandre Vranjac - Secretaria Estadual da Saúde de São Paulo. Foram selecionados nove municípios prioritários, considerandose três municípios de cada uma das seguintes regiões: Grande São Paulo, Baixada Santista/litorânea e Interior. Essas regiões apresentam diferenças em termos de incidência da TB (Grande São Paulo - 40,4/100.000 hab.; Baixada Santista - 94,9/100.000 hab.; Interior - 27,4/100.000 hab.)(11).

Os nove sujeitos dessa pesquisa foram os coordenadores do PCT dos respectivos municípios, selecionados por considerá-los fundamentais na identificação de dificuldades para incorporação das atividades técnicas da estratégia DOTS na ABS. 
A coleta de dados foi realizada em junho de 2005 através de entrevista semi-estruturada, contendo as seguintes questões norteadoras: como as equipes da ABS incorporam as atividades da estratégia DOTS para o controle da TB? Quais as barreiras para o desenvolvimento do DOTS na $A B S$ ? As entrevistas foram realizadas individualmente por profissionais de nível superior treinados. As falas foram gravadas e transcritas na íntegra com a autorização dos sujeitos participantes do estudo, mediante assinatura do Termo de Consentimento Livre e Esclarecido, em cumprimento da Resolução n. ${ }^{\circ} 196 / 96$ do Conselho Nacional de Saúde. Ressalta-se que este estudo está inserido em um projeto de âmbito nacional, intitulado: Implantação do DOTS em algumas regiões do Brasil: histórico e peculiaridades de acordo com as características regionais, aprovado pelo Comitê de Ética em Pesquisa da Escola de Enfermagem de Ribeirão Preto da Universidade de São Paulo (CEP - EERP-USP - 169/2005).

Para a análise dos dados, utilizou-se a técnica de análise de conteúdo, modalidade temática, que possibilitou organizar o conjunto das falas emitidas pelos coordenadores do PCT durante as entrevistas e descobrir os núcleos de sentido que compõem a comunicação cuja presença ou freqüência podem revelar aspectos significativos ${ }^{(12)}$ para a análise do envolvimento das equipes da ABS com as atividades da estratégia DOTS. Entende-se por envolvimento das equipes de saúde - a forma como incorporam e executam as atividades técnicas da estratégia DOTS, principalmente o TS e a BSR. A codificação dos depoimentos resultou na construção de dois núcleos de sentido: A debilidade quantitativa e qualitativa de RH na ABS; A visão centralizada e fragmentada da organização das ações de controle da TB no sistema de saúde. Estes núcleos de sentido permitiram a construção de uma Unidade Temática Central: A incorporação das ações de controle da TB na ABS.

\section{RESULTADOS E DISCUSSÃO}

Inicialmente destaca-se que nos municípios prioritários do Estado de São Paulo, algumas atividades, como o TS e a BRS estão sendo fortemente transferidas para os pontos periféricos do sistema municipal de saúde, principalmente para as UBSs ${ }^{(13)}$. No entanto, dificuldades foram identificadas em oito municípios para a incorporação de tais atividades pela ABS. A debilidade quantitativa e qualitativa de RH e a visão centralizada e fragmentada da organização das ações de controle da TB no sistema de saúde foram as principais barreiras apontadas nos depoimentos, as quais podem comprometer 0 acesso dos doentes às ações de diagnóstico e tratamento da TB, bem como a qualidade da interação profissional-usuário para 0 adequado manejo da doença na ABS.

Em relação à debilidade quantitativa e qualitativa de $\mathrm{RH}$ na ABS, presente em cinco depoimentos, esta se refere à falta de RH, sobrecarga de funções e inadequada qualificação dos profissionais das equipes de saúde para lidarem com a TB.
Identificou-se que profissionais de UBSs expressavam certa resistência à incorporação do TS e BSR na rotina diária das atividades executadas por esses serviços em decorrência da falta de RH.

As demais Unidades [UBS] ainda têm certa dificuldade de aceitar isso [TS]. Você sempre ouve [...] está faltando funcionário, não tem gente pra cuidar disso, não tem como dar prioridade ao paciente [...] (E9).

A dificuldade que eu vejo [BSR], é a do profissional [...] São coisas tão simples, mas a Unidade acaba colocando obstáculos pela falta de funcionários [...] (E9).

Essa situação faz com que os profissionais improvisem e executem suas tarefas em condições desfavoráveis, com possível prejuízo nas relações inter-pessoais e no desempenho dos serviços de saúde ${ }^{(14)}$. Na AB, a falta de RH pode resultar na sobrecarga de funções entre os profissionais e comprometer a capacidade resolutiva dos serviços, o processo de interação com o doente de TB, bem como o vínculo e adesão ao processo terapêutico. A sobrecarga de funções também pode ocorrer pela designação de múltiplas atividades, programas e tecnologias para ABS sem 0 adequado preparo e perfil profissional, dificultando a sensibilização e organização do processo de trabalho nesses serviços para incorporarem as ações de controle da TB.

\section{[...] na unidade básica já tem um monte de coisa e isto [TS] está sendo um grande mal na estrutura atual [...] o que tinha era o programa de pré-natal, puericultura e fazia vacinação, de repente, começou a receber clinica medica, programa do idoso [...] agora tuberculose [...] a estrutura não estava preparada, não tinha um $\mathrm{RH}$ suficiente e pre- parado (E2)}

A transferência de competências e responsabilidades para a ABS exige, além de adequada alocação de recursos, de um suporte gerencial e técnico para que UBS e PSF organizem suas práticas incorporando os princípios e atributos da Atenção Primária à Saúde para responderem às demandas da população de forma planejada, organizada, humanizada e resolutiva. Um sistema de saúde com base na Atenção Primária fundamenta-se no planejamento com intuito de oferecer recursos adequados, sustentáveis e apropriados às necessidades de saúde da população(15).

Outro aspecto que interfere na incorporação do controle da TB na ABS é a qualificação dos profissionais, uma vez que estes passaram a assumir ações e programas de saúde nunca antes implementados. Um dos depoimentos mostra que equipes de $A B$ têm dificuldade em realizar a BSR, pois não estão sensibilizadas e preparadas para detectar os sinais e sintomas da TB.

[...] quem tem que fazer a busca ativa é o profissional da área de saúde, desde a recepção até o médico [...] O pessoal [UBS] não incorpora que tosse há mais de três semanas pode ser tuberculose [...] se você não consegue por isso na cabeça do pessoal [...] quanto mais o resto [...] (E2). 
A capacitação em TB assume importante papel e deve garantir a compreensão da doença e seus condicionantes, bem como de instrumentos e tecnologias utilizadas para sua prevenção e controle, dentre elas a BSR e o TS. Supervisões contínuas também são necessárias para a identificação de falhas, dificuldades no processo de operacionalização do TS e da BSR, correções e aprimoramento de habilidades adquiridas. A prática da supervisão pautada na finalidade adminstrativo-gerencial deve ser superada, de modo que esta seja conduzida de forma motivadora(16) pelo coordenador do PCT para facilitar que as equipes da ABS se responsabilizem pelo controle da TB.

Um elemento que prejudica a manutenção de equipes de saúde qualificadas para lidar com a TB e que merece atenção dos responsáveis pela formulação de políticas de $\mathrm{RH}$ é a rotatividade dos profissionais, relacionada à descontinuidade político-partidária nos sistemas municipais de saúde, trazendo como conseqüência, a dificuldade para o estabelecimento de vínculo entre a equipe e os doentes.

\section{[...] se observa no PSF desse município uma grande rotatividade médica, então, treina, daqui a pouco você vai ver e já não é mais o médico que foi treinado [...] A enfer- magem também, não é que vai embora [...] mas roda muito entre os postos da cidade (E4). \\ [TB] Tem que ter vínculo [...] de 10 anos que estou aqui [...] está cheio de gente nova que mudou governo [...] (E2).}

Em relação à visão centralizada e fragmentada da organização das ações de controle da TB no sistema de saúde, dois depoimentos apontaram tal situação como um obstáculo para a incorporação do controle da doença na ABS. As atividades relacionadas à TB são consideradas pelas equipes de saúde como competência exclusiva dos centros de referência para tratamento da doença, o que contribui para a ausência de responsabilização local com 0 controle da mesma.

[...] a rede tinha aquela visão: tuberculose- centro de referência, então a partir daí eles perdiam o vínculo com esse paciente, achavam que não era problema mais deles [...] (E5).

[...] não acreditando [TS], não achando importante, acha que é uma coisa só do programa [...] que não é de todos (...) e não é verdade [...] É um programa que todos têm que estar participando (E9).

Tal situação indica que os serviços estão organizados na lógica dos sistemas fragmentados, cujo imperativo é a atenção aos eventos agudos, pois atuam através de pontos de atenção à saúde isolados e incomunicáveis entre si e são incapazes de prestar uma atenção contínua e se responsabilizarem por uma população determinada ${ }^{(17)}$. Para propiciar um ambiente favorável à prevenção e gerenciamento das ações de controle da TB na AB há que se avançar para um modelo de atenção integrado, voltado para as condições crônicas, tendo a Atenção Primária à Saúde como eixo norteador, porém sempre se partindo da premissa que a conscientização, o preparo e a motivação das equipes de saúde são fundamentais para o compromisso com o controle da doença. Destaca-se a relevância dos gerentes locais nesse processo, pois além das funções de planejamento, coordenação, direção e controle dos serviços para viabilizar políticas e alcançar objetivos e metas ${ }^{(14,18-19)}$, também são responsáveis pela mobilização e comprometimento dos profissionais na organização e produção de serviços/ações para atender às necessidades da população(14).

Cabe ressaltar que a questão da debilidade quantitativa e qualitativa de $\mathrm{RH}$ e a visão centralizada e fragmentada da organização das ações de controle da TB no sistema de saúde podem influenciar o envolvimento, bem como 0 desempenho das equipes da ABS na assistência aos doentes.

A dificuldade de envolvimento das equipes de saúde com as ações de controle da TB também foi identificada e esteve relacionada à percepção das mesmas, principalmente em relação ao TS, considerado além de trabalhoso, como um ato paternalista. Essa situação pode ser explicada em função do contexto desfavorável onde paira o despreparo das equipes da $A B S$, cujas práticas são operadas seguindo o modelo fragmentado que privilegia a atenção às condições agudas.

[incorporação TS] [...] Um pouco de relutância, tem os que
acham que é muito trabalhoso e que não dá certo (E3).

A dificuldade é as pessoas não entenderem o que é e porque ele [TS] é uma exigência hoje. Então tem isso, isso é paternalismo (E9).

Em decorrência da falta envolvimento profissional, situações de maus tratos, falta de respeito e humanização no atendimento aos doentes de TB podem ocorrer durante a realização do TS nas UBS.

[...] a família veio falar [...] eu não vou mais tomar remédio lá [UBS] [...] sou muito mal atendido, o pessoal me deixa esperando de duas a três horas para dar o remédio e quando dão o remédio falam: se vira para arrumar água (...) (E2).

[...] tuberculose, hanseníase, aids, o que a gente vê é uma falta de compromisso, uma falta realmente de profissionalismo (E9).

Para o controle da TB essa situação é preocupante, pois coloca em risco a adesão dos doentes ao tratamento medicamentoso. Esta adesão depende do vínculo e do acoIhimento proporcionado pelos profissionais de saúde ao doente durante a prática do $\mathrm{TS}^{(20)}$.

Alguns depoimentos apontaram maior envolvimento das equipes de PSF do que das UBS para a execução das atividades de controle da TB, principalmente o TS.

O profissional de saúde da UBS tem um menor compromisso do que o outro [ACS do PSF] [...] ele [UBS] ainda não se sensibilizou a essa estratégia (E7). 
[...] estamos tendo problemas nas UBS porque as enfermeiras e as auxiliares de enfermagem não estão aderindo adequadamente ao processo [...] começou a melhorar quando a gente passou para o ACS [...] dentro do pátio do PS (E2).

No entanto, o PSF ainda enfrenta dificuldades para organizar sua prática assistencial e incorporar novas atribuições, como o controle da TB.

\begin{abstract}
A teoria diz que o PSF é um programa estruturado para fazer atendimento no global, quando você começa a ver na prática, não é bem assim que funciona. Eles mesmos têm dificuldades, nos programas primários (...) quando você introduz mais um programa [TB] para eles [...] surgem os problemas $[\ldots]$ (E4).
\end{abstract}

Destaca-se que o contexto do depoimento E4 indica maior experiência (em relação a E7 e E2) no que tange à prática do PSF, pois além da maior cobertura populacional $(62,1 \%)$, que situa a estratégia no município em um processo de Transição Terminal(17), este também optou por descentralizar o próprio PCT para este Programa.

Nesse sentido, apesar da expectativa de que o PSF venha contribuir para o controle da TB, é possível inferir que a transferência de responsabilidades para esses pontos de atenção deve ser realizada de modo cauteloso e, sobretudo, gradual, uma vez que a realização das ações de controle não depende apenas de boa vontade e disposição, mas sim de equipes qualificadas para lidar com a complexidade da doença.

Este estudo também permitiu identificar estratégias que contribuem para o envolvimento das equipes de saúde com as ações de controle da TB, dentre elas a obrigatoriedade no preenchimento de relatórios mensais e encaminhamento para a coordenação do PCT.

[...] de um mês para cá, que a gente fez a implantação [da BSR] [...] [UBS] passaram a procurar mais [...] questionar mais [...] junto com a implantação do livro [de Sintomáticos Respiratórios] tem o boletim, que mensalmente eles vão ter que estar mandando para mim [...] então passou uma responsabilidade maior para eles [...] (E9).

0 envolvimento das equipes da ABS pode ser estimulado através de capacitações, supervisões contínuas pela

\section{REFERÊNCIAS}

1. World Health Organization (WHO). Tuberculosis control: surveillance, planning, financing: WHO Report 2006. Geneva; 2006.

2. Brasil. M inistério da Saúde. Portaria MS/GM n. 95, de 26 de janeiro de 2001. Divulga a Norma Operacional de Assistência à Saúde - NOAS-SUS 01/2001. Diário Oficial da União, Brasília, 29 jan. 2001. Seção 1, p. 23E-31E.

3. Brasil. Ministério da Saúde. Fundação Nacional de Saúde. Plano Nacional de Controle da Tuberculose. Brasília; 2004. coordenação do PCT e estabelecimento de um fluxo de informações que garanta, tanto a qualidade/fidedignidade dos dados como o feedback dos resultados produzidos pelos serviços de saúde da $A B$, valorizando assim, o trabalho dos profissionais que aí atuam.

Para que as ações de controle da TB sejam incorporadas de forma expressiva, consciente e responsável no elenco de atividades dos serviços de ABS, há que se considerar que esse processo deve ser gradual e exige a elaboração de um projeto colaborativo, envolvendo coordenadores do PCT, do PSF/PACS, gerentes de UBS e representantes das equipes de PSF para definição conjunta de prioridades e estratégias de atuação, bem como para designação de atribuições e responsabilidades conforme a capacidade gerencial e técnica local.

\section{CONSIDERAÇÕES FINAIS}

O estudo apontou o descompasso entre a transferência de responsabilidades das ações de controle da TB para a $A B S$ e a inadequação quantitativa e qualitativa de RH para o manejo da doença no sistema de saúde. Os desafios para a incorporação da BSR e TS na ABS incluem, além do avanço na organização de um sistema integrado de saúde que contemple os princípios da Atenção Primária à Saúde para lidar com as condições crônicas, da elaboração e implementação de uma efetiva política de RH que garanta a formação e capacitação contínua dos profissionais. Destaca-se o papel essencial da coordenação do PCT no incentivo, organização, viabilização, supervisão contínua e monitoramento da situação da TB para a reorganização da atenção à doença no contexto da ABS. É necessária a designação de profissionais capacitados e com transito político para assumirem o gerenciamento e condução desse processo no nível municipal.

Enfim, para a viabilização das ações de controle da TB na ABS é fundamental a conscientização, o envolvimento, a integração e a articulação permanente dos responsáveis pelo controle da doença nos diversos níveis do sistema de saúde para a viabilização de políticas, planejamento, avaliação e adequação em conjunto das estratégias e tecnologias adotadas principalmente no nível municipal, que é onde ocorre de fato a implementação das políticas.

4. Beaglehole R, Bonita R, Horton R, Adams 0 , Mckee M. Public health in the new era: improving health through collective action. Lancet. 2004;363(9426):2084-6.

5. Organização Mundial de Saúde (OMS). Cuidados inovadores para condições crônicas: componentes estruturais de ação: relatório mundial. Brasília; 2003.

6. Cornetta VK, Maia CCA, Costa WGA. A reorganização dos serviços de saúde no sistema único de saúde e a formação de recursos humanos. Saúde Debate. 1996;(2):44-9. 
7. Figueroa-M uñoz J, Palmer K, Poz MR, Blanc L, Bergström K, Raviglione $M$. The health workforce crisis in TB control: a report from high-burden countries. Hum Resour Health [serial on the Internet]. 2005 [cited 2007 Jan 7];3(2): [about 9 p.] Available from: http://www.humanresources-health.com/content/3/1/2/abstract

8. World Health Organization (WHO). Tuberculosis control: surveillance, planning, financing: WHO Report 2003. Geneva; 2003.

9. World Health Organization (WHO). Tuberculosis control: surveillance, planning, financing: WHO Report 2004. Geneva; 2004.

10. World Health Organization (WHO). Tuberculosis control: surveillance, planning, financing: WHO Report 2005. Geneva; 2005.

11. São Paulo (Estado). Secretaria de Estado da Saúde. Centro de Vigilância Epidemiológica. Tuberculose. Indicadores de morbimortalidade e indicadores de desempenho. Bol Epidemiol Paul. 2006;3 Supl 4:7-37.

12. Bardin L. Análise de conteúdo. Lisboa: Edições 70; 1979.

13. Villa TCS, Monroe AA, Gonzales-Cardozo RI, Arcêncio RA, Oliveira MF, Galesi VMN, et al.A experiência da implantação da estratégia DOTS no estado de São Paulo. In: Ruffino Netto A, Villa, TCS. Tuberculose: implantação do DOTS em algumas regiões do Brasil: histórico e peculiaridades regionais. Ribeirão Preto: FM RP/USP; 2006. p. $75-80$.
14. Junqueira LAP. Gerência dos serviços de saúde. Cad Saúde Pública. 1990;6(3):247-59.

15. Organização Pan-Americana da Saúde (OPAS). Renovação da atenção primária em saúde nas Américas: documento de posicionamento da Organização PanAmericana da Saúde/OPAS [texto na Internet]. 2005. [citado 2006 nov. 06] Disponível em: www.paho.org/ portuguese/ad/ths/os/phc2ppaper_10-ago-05_Por.pdf

16. Fortuna CM, Mishima SM, Matumoto S, Domingos NAM Supervisão de equipes no Programa de Saúde da Família: reflexões acerca do desafio da produção de cuidados. Interface Comunic Saúde Educ. 2005;9(16):9-24.

17. Mendes EV. A atenção primária à saúde no SUS. Fortaleza: Escola de Saúde Pública do Ceará, Universidade Federal do Ceará; 2002.

18. Passos JP, Ciosak SI. A concepção dos enfermeiros no processo gerencial em Unidade Básica de Saúde. Rev Esc Enferm USP. 2006;40(4):464-8.

19. Ferreira AS. Competências gerenciais para Unidades Básicas do Sistema Único de Saúde. Ciênc Saúde Coletiva. 2004;9(1):69-9.

20. Bertollozi MR. A adesão ao tratamento da tuberculose na perspectiva da estratégia do tratamento diretamente observado (DOTS) no município de São Paulo-SP [tese livre-docência]. São Paulo: Escola de Enfermagem, Universidade de São Paulo; 2005.

\section{AGRADEaMENIOS}

Ao Conselho Nacional de Desenvolvimento Científico e Tecnológico à Pesquisa (CNPq), pelo apoio financeiro (Bolsa Doutorado, processo n 141908/03-3).

À Fundação de Apoio à Pesquisa do Estado de São Paulo (FAPESP) - Projeto Temático (processo n. ${ }^{\circ}$ 2003/08386-3), pelo apoio financeiros para assessoria durante a análise dos dados.

Ao Grupo de Estudos Operacionais em Tuberculose da REDE-TB e à Divisão de Tuberculose do Centro de Vigilância Epidemiológica Prof. Alexandre Vranjac-SES/SP pela colaboração durante a coleta e discussão dos dados. 